\title{
Salud y desarrollo de adolescentes y jóvenes en Latinoamérica y El Caribe: desafíos para la próxima década
}

\author{
Matilde Maddaleno, MD, MPH, (1) Paola Morello, MD, MPH, (2) \\ Francisca Infante-Espínola, Ed M.(3)
}

\section{Maddaleno M, Morello P, Infante-Espínola F. Salud y desarrollo de adolescentes y jóvenes en Latinoamérica y El Caribe: desafíos para la próxima década. \\ Salud Publica Mex 2003;45 supl 1:S132-S139.}

\section{Resumen}

Adolescentes y jóvenes representan $30 \%$ de la población de las Américas. Su salud es clave para el progreso social, económico y político de la Región. Sin embargo, con demasiada frecuencia sus necesidades no figuran en la agenda pública ni política y los go biernos no consideran prio ritario invertir en ellos. La O rganización Panamericana de la Salud (O PS) propone un nuevo marco conceptual centrado en el desarrollo humano y en la promoción de la salud dentro del contexto de la familia, la comunidad y el desarrollo social, político y económico. El gran desafío de los próximos años será utilizar este marco para implantar programas integrales, recolectar información desagregada, mejorar el acceso a los ser vicios, el ambiente donde viven adolescentes y jóvenes, el vínculo entre escuelas, familias y comunidades, así como apoyar la transición a la edad adulta, con una amplia participación juvenil y coordinación interinstitucional e intersectorial.

Palabras clave: ado lescencia; desarrollo integral; salud; políticas de salud;A mérica Latina

\author{
Maddaleno M, Morello P, Infante-Espínola F. \\ Health and development of adolescents and young adults \\ in Latin America and the Caribbean: challenges \\ for the next decade. \\ Salud Publica Mex 2003;45 suppl 1:S132-S139.
}

\section{A bstract}

Adolescents and young adults make up $30 \%$ of the population of the A mericas. Their health is a key factor in the social, economic, and political development of the region. $\mathrm{N}$ evertheless, their needs are frequently excluded from governments' public and political agendas. The Pan American Health $\mathrm{O}$ rganization (PAHO) advances a new conceptual framework focusing on human develo pment and health promotion within the context of family and community, and of social, political, and economic develo pment. The challenge in the near future is to use this framework for establishing comprehensive programs, collect disaggregated data, improve access to services, adolescents' environs, the ties between schools, families and communities, as well as improve and support the transition to adulthood through youth participation and interinstitutional and intersectoral collaboration.

Key words: adolescence; comprehensive development; health; health policies; Latin America

(1) Asesora Regional en Salud y D esarrollo de A dolescentes y Jóvenes. O rganización Panamericana de la Salud (0 PS), 0 rganización Mundial de la Salud (OMS).

(2) Consultora Programa de Salud y Desarrollo de Adolescentes y Jóvenes. O PS, 0 MS.

(3) Psicóloga especialista en D esarrollo Adolescente. O PS, O MS. 
a salud de adolescentes (10 a 19 años de edad) y de jóvenes ( 15 a 24 años de edad) es un elemento clave para el progreso social, económico y político de todos los países y territorios de las Américas. Sin embargo, con demasiada frecuencia las necesidades y los derechos de las y los adolescentes no figuran en las políticas públicas, ni en la agenda del sector salud, excepto cuando su conducta es inadecuada. Uno de los factores que contribuye a esta omisión es que éstos en comparación con los niños y los adultos mayores, sufren de pocas enfermedades que ponen en riesgo sus vidas. ${ }^{1}$ Sin embargo, aunque gran parte de los hábitos nocivos para la salud adquiridos durante la adolescencia no se manifiestan en morbilidad o mortalidad durante la adolescencia misma, sí lo hacen en años posteriores. ${ }^{1}$ De hecho, la Organización Mundial de la Salud (OMS) estima que $70 \%$ de las muertes prematuras en el adulto se deben a conductas iniciadas en la adolescencia.

Actualmente disponemos de amplia información y experiencia sobre la estructuración de planes, programas y servicios para justificar la inversión en este grupo etáreo. Si bien el objetivo de esta inversión es contribuir al bienestar de los jóvenes en general, hay que recordar que no se puede considerar a la "juventud" como una categoría homogénea. La población joven es un conjunto heterogéneo y diverso, con múltiples identidades de género, cultura, etnia, estatus social y económico, vida urbana y rural, las cuales merecen ser exploradas.

$\mathrm{Al}$ igual que se habla de "juventudes" en todas sus variantes, no hay que limitarse a tratar a adolescentes y jóvenes cuando ya exhiben comportamientos indeseados; se debe trabajar dentro de un contexto más amplio de promoción de salud y desarrollo humano. El desarrollo se define como un proceso continuo a través del cual satisfacen sus necesidades y desarrollan competencias, habilidades y redes sociales. Para conseguir un desarrollo pleno, son claves el acceso a la salud y el bienestar, la educación, la justicia, el empleo y la participación social, además del apoyo a las familias y comunidades para que guíen el desarrollo de los jóvenes. ${ }^{1}$ La información recolectada hasta el momento servirá para desbancar mitos y desacuerdos institucionales que impiden la promoción de la salud y el desarrollo de adolescentes y jóvenes.

\section{Situación actual de salud de adolescentes y jóvenes en Latinoamérica y El Caribe}

Se estima que entre 1960 y 1990, el número de adolescentes de 10 a 19 años de edad aumentó 138\% en la
Región. ${ }^{2}$ Datos de la Comisión Económica para América Latina (Cepal) estiman que en 1999, la población de 10 a 19 años representaba $21 \%$ de la población total de la Región, siendo la distribución homogénea por sexo. ${ }^{3}$ Por otra parte, se estima que en el año $2000,80 \%$ de la población joven (10 a 24 años) vive en áreas urbanas. ${ }^{4} \mathrm{El}$ deterioro de las condiciones de vida de la población ha afectado en mayor medida los hogares donde existen niños y adolescentes tanto en áreas urbanas como rurales. Se estima que la pobreza en el año 2000 alcanza $56 \%$ de los niños y adolescentes de 0 a 19 años de edad en América Latina. ${ }^{5}$

\section{Educación}

El nivel de educación de la juventud de Latinoamérica y El Caribe (LAC) ha experimentado una mejora dramática en las últimas décadas. En 1970, 26\% de la población de más de 15 años de edad era analfabeta; se estima que sólo $12 \%$ lo es en el año $2000 .{ }^{6}$ Los porcentajes de analfabetismo en jóvenes de 15 a 19 años de la Región fluctúan entre 12.4 a $0 \%$ con un promedio de $2.3 \%$ para la Región. ${ }^{7}$ Además, jóvenes de esta generación están completando más años de educación que sus padres. Aun así, sólo jóvenes del área urbana de Chile completan los 12 años de educación necesarios para superar la pobreza durante la vida activa. ${ }^{8}$ En la década de los 90, Chile, Honduras y México disminuyeron de manera considerable las disparidades urbano/rurales. Es interesante notar que en El Salvador, $60 \%$ de los niños y adolescentes que no van a la escuela provienen de áreas rurales. ${ }^{9}$

En una encuesta realizada en 15 países de la Región se evidenció que sólo 7\% de escolares que provienen de familias en el cuartil más alto de ingreso y $26 \%$ de los que provienen de familias de los cuartiles más bajos abandonan la escuela. ${ }^{5}$ En México, por ejemplo, existe una gran variabilidad dentro del propio país, donde $80 \%$ de jóvenes de clase alta y sólo $18 \%$ de la clase baja han completado al menos 10 años de estudio. ${ }^{10}$

Se estimó que para el año 2000, cerca de la mitad de la población de 20 años de edad habría abandonado los estudios sin terminar la secundaria; los porcentajes son mayores en las familias de menores ingresos. Sin embargo, en la mayoría de los países de la Región al menos uno de cada 10 jóvenes comienza una carrera universitaria. ${ }^{5}$

La mayoría de los países ha mejorado el acceso a la educación básica para las mujeres. Un buen ejemplo es que las mujeres en la Región tienen una mayor probabilidad de asistir a la escuela con los varones de su misma edad que en otros países en desarrollo. ${ }^{11}$ 


\section{Empleo}

Entre 1997 y 1999, el desempleo urbano en América Latina aumentó de 7.3 a $8.7 \%$ en la población activa. ${ }^{5}$ Jóvenes de 15 a 19 años de edad representan entre 40 y $50 \%$ de la población económicamente activa. ${ }^{4}$ En El Salvador, adolescentes de 10 a 17 años representan 16\% de la fuerza laboral del país; de esos, 71\% son varones y $78 \%$ está en el área rural. ${ }^{9}$ Las tasas de desempleo en jóvenes siguen siendo desalentadoras: varían entre 36 y 66\%. ${ }^{9}$ También aquí vemos una gran variabilidad entre hombres y mujeres, y entre los distintos niveles socioeconómicos, ya que las tasas de desempleo son mayores en mujeres, en pobres y en los grupos con menor nivel de educación. ${ }^{4}$ Encuestas realizadas en 15 países de la Región muestran que 12 a 40\% de jóvenes de clase baja y sólo 2 a 10\% de clase alta no estudia ni trabaja. ${ }^{3}$

Por otro lado, se estima que existen aproximadamente 10 millones de niños y niñas menores de 14 años de edad que trabajan ilegalmente sin ninguna clase de beneficios y bajo condiciones de trabajo inadecuadas. ${ }^{4}$

\section{Familia}

El 20\% de los hogares de América Latina y El Caribe tienen hijos de 13 a 18 años de edad (17\% en Chile y Uruguay; 23\% en Honduras y Bolivia). ${ }^{12}$

Se ha demostrado que la incidencia de pobreza es mayor en los hogares encabezados por mujeres. Cepal ha estimado que, desde 1994, aproximadamente uno de cada cinco hogares en América Latina está encabezado por una mujer. En Costa Rica el porcentaje de hogares encabezado por una mujer (1998) es de $21 \%$, en El Salvador de 35\%* y en Trinidad y Tobago, 25\% (32\% en centros urbanos). ${ }^{14}$

La mitad de adolescentes encuestados en nueve países de El Caribe vive en un hogar con dos padres. Sin embargo, la unidad familiar en El Caribe ha cambiado, y existen nuevas categorías de familia como por ejemplo las "relaciones de visitante", donde uno de los miembros de la pareja, generalmente el hombre, visita a la pareja periódicamente. ${ }^{11}$

\section{Salud general}

Más de $80 \%$ de escolares de 10 a 18 años de edad encuestados en nueve países de El Caribe describió su

\footnotetext{
* Núñez JG, Martínez MA, Maynard-Tucker G, Murray N. Proyecto de servicios integrales para adolescentes en condiciones de pobreza. Informe final. Futures Group International, Banco Interamericano de Desarrollo/OPS/Paniamor de Costa Rica. Informe no publicado. Washington, D.C, mayo 2000.
}

salud como buena o excelente. La existencia de una buena comunicación familiar se identificó como un factor de protección de la salud en general. Así, el riesgo asociado con tener problemas de salud era aproximadamente $50 \%$ menor en quienes se sentían conectados con su familia. Los problemas de salud que adolescentes de El Caribe identificaron con mayor frecuencia fueron cefaleas, trastornos del crecimiento y trastornos del sueño, asociados en algunos casos con problemas familiares y abuso. Casi 70\% de los encuestados estaba conforme con su peso y más de $75 \%$ dijo hacer algún tipo de actividad física al menos una vez por semana. ${ }^{15}$

Las tasas de mortalidad de adolescentes y jóvenes son bajas en comparación con los otros grupos etáreos y la mayoría de las muertes son debidas a causas externas (accidentes, homicidios y suicidios). En la mayoría de los países, los accidentes de tráfico son la causa más importante de mortalidad en este grupo etáreo. ${ }^{4}$

\section{Salud sexual y reproductiva}

Existe un aumento en la actividad sexual de jóvenes a edades cada vez más tempranas, y la tasa de fertilidad en mujeres adolescentes es mayor a 50 por 1000 en varios países. La figura 1 refleja la inequidad en la tasa de fertilidad específica entre los 15 y 19 años de edad según los quintiles de ingreso, evidenciando que es entre los pobres donde persisten los mayores problemas.

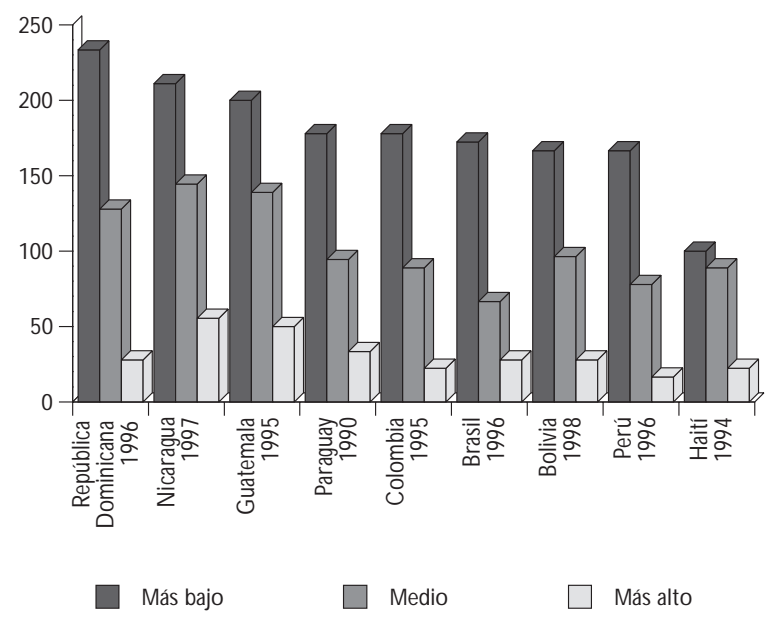

Fuente: Banco Mundial, Poverty Inequity Project

Figura 1.TASA de fertilidad específica (15-19 años) POR QUINTILES EN PAÍSES SELECCIONADOS DE LATINOAMÉRICA y EL CARIBE, 1990-1998 


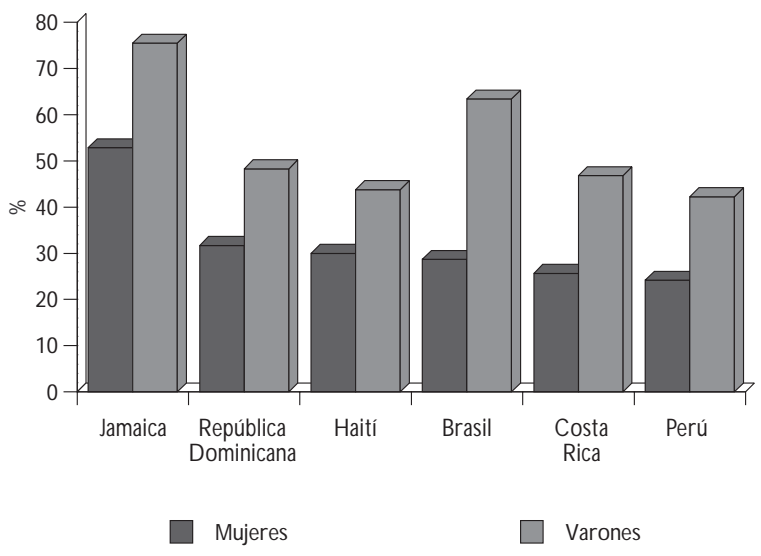

Fuente: Singh y cols., International Family Perspectives, 2000

Figura 2. Porcentaje de jóvenes (20-24 años) que INICIA RELACIONES SEXUALES ANTES DE LOS 17 AÑOS DE EDAD EN PAíSES SELECCIONADOS DE LATINOAMÉRICA Y EL Caribe, 2000

Se estima que $50 \%$ de las mujeres de LAC ha tenido una relación sexual antes de los 20 años de edad. ${ }^{2}$ La figura 2 muestra el porcentaje de jóvenes de 20 a 24 años de edad que tuvo una relación sexual antes de los 17 años en seis países de la Región. ${ }^{16}$ En una muestra representativa de adolescentes escolarizados de nueve países de El Caribe, un tercio dijo haber tenido relaciones sexuales, de las cuales $50 \%$ había sido bajo coerción. ${ }^{15}$ Durante 1999, una de cada 12 mujeres adolescentes nicaragüenses en unión había sido víctima de violencia sexual. ${ }^{19}$

En la encuesta realizada en escolares de 10 a 18 años de edad en nueve países de El Caribe, se indagó sobre la orientación sexual. Aproximadamente 5\% de los encuestados dijo sentirse atraído únicamente por personas de su mismo sexo. Este porcentaje fue levemente superior en los hombres y disminuyó con la edad. ${ }^{15}$

En cuanto a la anticoncepción, la mayor parte de jóvenes conoce los métodos. Sin embargo, la situación no es óptima: el uso de anticonceptivos durante la primera relación sexual en mujeres menores de 18 años de edad es bajo, variando entre $23 \%$ en El Salvador y $10 \%$ en Guatemala, Paraguay y Panamá. Aun así, tanto en las mujeres como en los hombres, el uso de anticonceptivos aumenta con la edad. ${ }^{2}$ Se ha demostrado que las adolescentes de bajos recursos tienen más riesgo de embarazo ( $80 \%$ en área urbana y $70 \%$ en área rural). ${ }^{8}$ Cerca de un tercio de las mujeres entre 20 y 24 años de edad en 10 países de LAC ha tenido un hijo antes de los 20 años. ${ }^{2}$ Durante 1999, 35\% de los partos atendidos por los servicios del Ministerio de Salud de El Salvador fueron de adolescentes. ${ }^{9}$

Las infecciones de transmisión sexual afectan a $15 \%$ de adolescentes de 15 a 19 años de edad, quienes adquieren cada año tricomoniasis, Chlamydia, gonorrea o sífilis. ${ }^{2}$ La mitad de los casos nuevos del VIH ocurren en jóvenes de 15 a 24 años, y la edad promedio de casos está disminuyendo: entre 1983 y 1989 era de 32 años; de 1990 a 92 bajó a 25 años. ${ }^{4}$ Se estima que en Haití, República Dominicana, Honduras, Panamá y Guatemala, más de 1\% de la población de 15 a 24 años de edad es seropositiva (cuadro I). Por ejemplo, en Haití, se estima que $4.9 \%$ de los hombres y $2.9 \%$ de las mujeres de 15 a 24 años de edad son seropositivas. Estos datos contrastan con otras regiones del mundo, como en Africa, donde la infección con el VIH es mayor en jóvenes mujeres que en varones. ${ }^{17,14}$ En países como Nicaragua, Brasil o Perú, 27 a 33\% de las mujeres adolescentes no conoce ningún método para protegerse del VIH. El porcentaje de hombres que desconoce es menor pero sigue siendo elevado, entre 15 y $26 \%{ }^{18}$ En El Salvador, $18.2 \%$ de los casos de VIH se encuentra en jóvenes de 12 a 24 años de edad; $90 \%$ reportó que la vía de transmisión más frecuente fue a través de las relaciones sexuales. ${ }^{9}$ En Haití, $63 \%$ de las mujeres de 15 a 19 años de edad sexualmente activas piensa que no tiene ningún riesgo de contraer el VIH. Este porcentaje es significativamente mayor que en varios países de Africa (Uganda, 21\%; Kenya, 36\% y Camerún, 43\%).

\section{Abuso de sustancias}

La edad de inicio de consumo de drogas ilícitas se encuentra en los 13 años. ${ }^{19}$ Sin embargo, en algunos países se encuentra en descenso: en Costa Rica la edad de inicio de consumo de tabaco era de 11 años en 1999. ${ }^{20}$ En los países de El Caribe, el alcohol es la principal droga de consumo; $54 \%$ de adolescentes encuestados y $40 \%$ de las adolescentes había consumido alcohol

Cuadro I

Estimación del PORCENTAJe de Jóvenes DE 15 A 24 AÑOS INFECTAdOS CON VIH, 1999

Mujeres Hombres

\begin{tabular}{lll} 
Haití & 2.9 & 4.9 \\
\hline República D ominicana & 2.8 & 2.6 \\
\hline Honduras & 1.7 & 1.4 \\
\hline Panamá & 1.4 & 1.7 \\
\hline Guatemala & 0.9 & 1.2
\end{tabular}

Fuente:UN AID S/U N IC EF citado enThe Progress of N ations 2000.U N ICEF, 2000 
alguna vez en su vida. ${ }^{14}$ Aunque el consumo de tabaco es escaso en El Caribe, países como Perú o Cuba tienen índices más altos. ${ }^{10}$ Por otra parte, en Argentina, se hizo en 1997 una encuesta en alumnos de 12-18 años de edad demostrando que 30\% había fumado un cigarrillo en los 30 días previos a la encuesta, siendo similar el consumo en ambos sexos. ${ }^{21}$

\section{Salud mental}

Entre 11 y 33\% de jóvenes de la Región refiere tener algún problema de salud mental. Es difícil establecer comparaciones entre los países ya que las encuestas utilizadas difieren de país a país. Los síntomas depresivos y las conductas suicidas son los más frecuentes. Canadá, Cuba, El Salvador, Trinidad y Tobago, Estados Unidos de América (EUA), Uruguay y Venezuela tienen tasas de suicidio en varones de 15 a 24 años de edad que superan los 10 por 100.000 habitantes. ${ }^{19}$ En una encuesta de adolescentes escolarizados de nueve países de El Caribe se evidenció que 12\% de los encuestados había intentado suicidarse y que 50\% había tenido síntomas de depresión. ${ }^{15}$ En casi todos los países, las tasas de suicidio de los varones son el doble que las de las mujeres. ${ }^{19}$

El problema de la violencia se agrava cada vez más en la Región. El 29\% de las muertes por homicidio en la Región fueron en adolescentes de 10 a 19 años de edad. El caso de Colombia es aún más dramático: 15\% de las víctimas mortales del total de actos violentos y $20 \%$ de los homicidios de toda la Región ocurren en este país. En Nicaragua existen alrededor de 156 grupos pandilleros (maras) de los cuales $69 \%$ se ubican en la capital. ${ }^{9}$ Un alto porcentaje de adolescentes $(7 \%$ de las mujeres y $20 \%$ de los hombres) había portado un arma en la escuela durante los 30 días previos a la encuesta en los países de El Caribe. ${ }^{9}$

\section{Servicios}

En una encuesta efectuada en nueve países de El Caribe, $86 \%$ de adolescentes pudo identificar un lugar de atención sanitaria, pero sólo $36 \%$ refirió haber visto a un médico en los últimos dos años. Por otro lado, menos de $50 \%$ consultó a un dentista en los últimos dos años. De hecho, datos de la Organización Panamericana de la Salud muestran que el porcentaje de CPO (caries, pérdidas y obturaciones) a los 12 años de edad llega a 1.1 en Jamaica, 4.4 en República Dominicana y 4.7 en Bolivia. ${ }^{22}$ Un estudio realizado en adolescentes varones de nueve países de la Región demostró que los mismos formulan el deseo de tener servicios de salud de calidad con espacios dirigidos específicamente a ellos, que les garanticen confidencialidad, privacidad y que no sean condescendientes. ${ }^{23}$

A pesar de la situación descrita de salud y desarrollo, la adolescencia y la juventud aún no figuran como un área prioritaria en la agenda pública.

\section{¿Por qué no se invierte suficiente en salud y desarrollo?}

Aunque jóvenes y adolescentes representan un potencial para el desarrollo social y económico de la Región, los gobiernos de muchos países no consideran prioritario invertir en ellos. Esto se debe en parte a que la información existente sobre adolescentes y jóvenes es escasa, y los pocos datos que existen se centran en conductas problema o de riesgo y no están desagregados por edad o sexo. Esta carencia dificulta la realización de estudios comparativos entre comunidades, países o subregiones y las actividades de abogacía específicas para este grupo etáreo.

En comparación con los niños y los adultos mayores los adolescentes jóvenes presentan menos morbimortalidad, fomentando así el mito de que éstos no se enferman. Sin embargo, no se tiene en cuenta, por ejemplo, que adolescentes y jóvenes están afectados por problemas de salud sexual y reproductiva y otras patologías, y que las conductas iniciadas en la adolescencia tienen consecuencias futuras en términos de morbilidad.

La inversión es difícil al no haber consenso en la sociedad en cuanto a temas altamente sensibles, como la conducta sexual de adolescentes o el consumo de sustancias, y los gobiernos se muestran ambivalentes respecto del papel que debe tomar el Estado con relación a éstas y otras conductas juveniles.

También existe el mito de que no hay evidencia científica sobre cómo tratar los problemas de jóvenes adolescentes y; sin embargo, en los últimos 10 años se ha generado suficiente conocimiento científico en el tema de promoción o prevención.

Apoyar el desarrollo de adolescentes y jóvenes requiere de una inversión a largo plazo y no de intervenciones aisladas, de corto plazo, sin impacto y sin continuidad. El tema se complica más al comprobar que adolescentes y jóvenes son invisibles en algunos países, y dirigirse a un problema invisible requiere visión de futuro, determinación y sabiduría. Esto adquiere especial relevancia cuando el tema de adolescencia y juventud compite por atención y financiamiento con otros problemas de salud, como la malaria, la tuberculosis o la mortalidad infantil. 


\section{¿Por qué debemos invertir en salud y desarrollo de adolescentes?}

Jóvenes (10 a 24 años de edad) representan 30\% de la población en la Región, y el porcentaje mayor está concentrado en los países más pobres. ${ }^{4}$ Hoy son los líderes del nuevo milenio, e invertir en la juventud contribuirá a la salud general, la educación y las condiciones sociales de las presentes y futuras generaciones. Algunos países están en plena transición demográfica, con posibilidades de disfrutar del denominado "regalo demográfico". En los países en desarrollo las menores tasas de natalidad y el hecho de que aún no se cuenta con un contingente significativo de población anciana ofrecen la posibilidad de un dividendo demográfico en los próximos 15 a 20 años. De esta manera, si los gobiernos invierten ahora en salud y educación secundaria universal para sus jóvenes, cuando éstos se integren a la fuerza laboral, su productividad contribuirá al incremento significativo de los niveles del Producto Global Interno, ${ }^{24}$ como ocurrió en los países del este asiático.

Además de aportar mayores perspectivas de futuro, invertir en promoción y prevención puede significar un ahorro para el país. ${ }^{10}$ Un reciente análisis de costo-beneficio estimó que cada año en EUA se gastan 20 billones de dólares para mantener los ingresos, servicios de salud y nutrición de las familias encabezadas por adolescentes. Por otra parte, el costo de un drogadicto o de un delincuente reincidente varía entre 1 y 1.3 millones. Se estima que estos gastos pueden reducirse a la mitad si se invierte en prevención. ${ }^{10}$ Aparte del ahorro que supone la prevención, hay que recordar que la salud de adolescentes y jóvenes es un derecho humano, como aprobaron los gobiernos en la Cumbre de la Infancia de 1990. Invertir en la salud de adolescentes implica potenciar la inversión previa de los gobiernos en supervivencia infantil, prevención de enfermedades infecciosas y educación.

Tampoco hay que olvidar el impacto directo que tiene la salud en el potencial educativo de adolescentes y jóvenes, y viceversa. La salud es un factor indispensable para la sustentabilidad del desarrollo, ya que las buenas condiciones físicas y mentales contribuyen a mejorar y potenciar el rendimiento escolar. Se estima que un año de educación puede reducir la fertilidad de las adolescentes entre 5 y $10 \%$. Las adolescentes que se quedan embarazadas terminan con menos educación y con un mayor nivel de pobreza al abandonar el sistema educativo. ${ }^{25}$

\section{Desafíos en salud y desarrollo de adolescentes para la próxima década}

Tradicionalmente, los programas de salud de adolescentes en Latinoamérica han centrado sus esfuerzos en jóvenes que ya demuestran comportamientos indeseados por la sociedad; por ejemplo, deserción escolar, violencia juvenil, abuso de drogas ilícitas o delincuencia. Los programas que tratan esos problemas intentan, generalmente, convencerles de que abandonen esos comportamientos para reducir el impacto de sus consecuencias. Así, la mayoría se centran en la prevención secundaria y terciaria, y no en un enfoque de promoción de salud y resiliencia y conductas positivas. Actualmente, disponemos de abundante información que demuestra que los programas de atención secundaria o terciaria no logran una reducción significativa en los comportamientos negativos juveniles, ya que se centran en el individuo y no tienen en cuenta la influencia de la familia, el medio ambiente y el contexto general en el que ocurren estos comportamientos. Basándose en esta información, la unidad técnica de salud y desarrollo adolescente de la Organización Panamericana de la Salud (OPS) ha propuesto un marco conceptual que se centra en el desarrollo humano y en la promoción de la salud dentro del contexto de la familia, la comunidad y el desarrollo social, político y económico (figura 3).

Se sabe que el enfoque integral de desarrollo humano propuesto en el marco conceptual de la OPS es una estrategia de promoción de salud, y que invertir en adolescentes supone un ahorro futuro para los países. De esta manera, el gran desafío de los próximos años será utilizar este marco para desarrollar programas integrales, recolectar información desagregada, mejorar el acceso a los servicios, mejorar el ambiente donde viven, comprometerse con la promoción de salud, mejorar el vínculo entre escuelas, familias y comunidades, apoyar la transición a la edad adulta, utilizar un enfoque de género en los servicios, fomentar la participación juvenil y promover la articulación interinstitucional. Todas estas recomendaciones se describen a continuación. ${ }^{26}$

1. Incrementar el acceso y la producción de información respecto a adolescentes. Los países y los ministerios de salud, educación y juventud deben continuar recolectando información básica sobre la situación del desarrollo integral de adolescentes y jóvenes. Para la próxima década se deberá recolectar, además de los indicadores clásicos de salud, otros indicadores de desarrollo y resiliencia, como por ejemplo, cuántos adolescentes se sienten seguros en su entorno, cuántos adolescentes están conformes con su imagen corporal, cuántas adolescentes se quedan embarazadas, cuántos jóvenes son violentos o participan en pandillas juveniles, y qué perspectivas de futuro tienen. Esta información debería estar desagregada por grupos de 


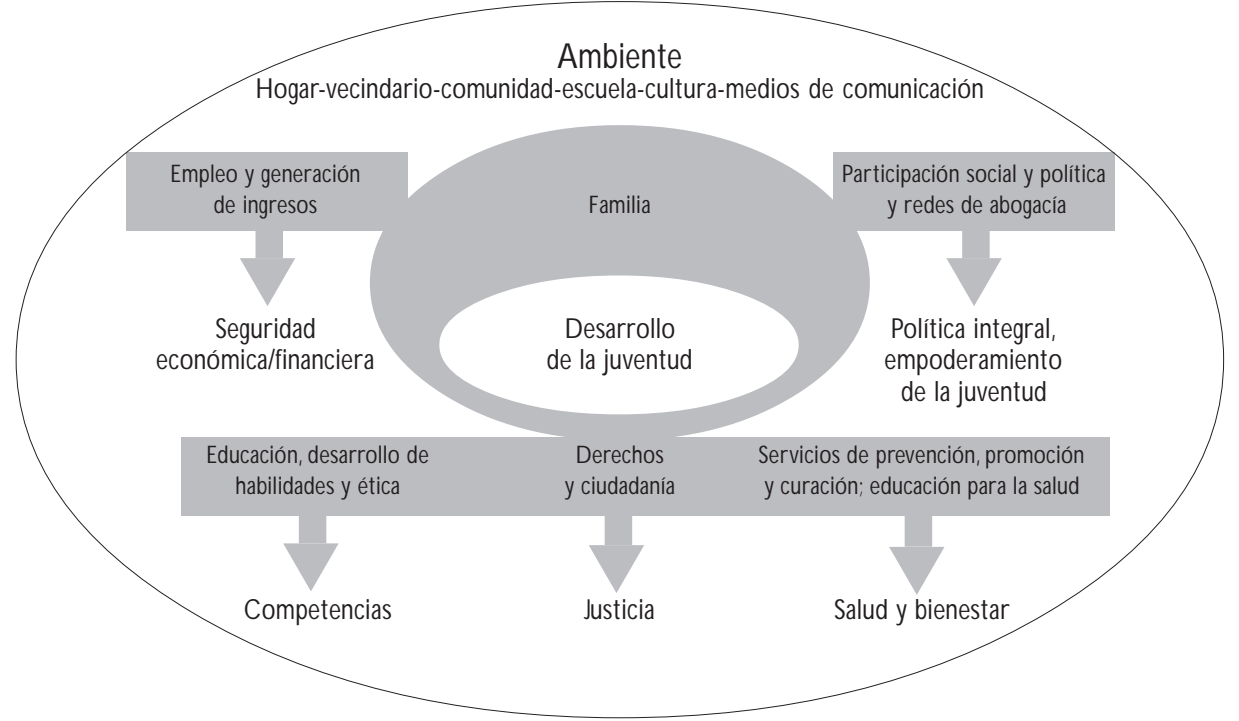

Fuente: Plan de acción de desarrollo y salud de adolescentes y jóvenes en las A méricas 1998-2001. O PS, 1998

Figura 3. Modelo para promover la salud y el desarrollo de adolescentes y jóvenes de Latinoamérica y El CARIBE

edad, género, urbano/rural, etcétera. Así, podrá servir como herramienta de abogacía para influir en el diseño e implantación de políticas públicas, planes y programas, y para desmentir mitos que perjudican a adolescentes y jóvenes. En la próxima década también se deberán documentar aquellas experiencias que son eficaces y efectivas, y abandonar aquellas que no sirven en la promoción del desarrollo y la salud de adolescentes.

2. Mejorar el acceso a los servicios de salud. Los países que están reformando el sector salud deben asegurar el acceso equitativo de todo adolescente a un paquete mínimo de servicios integrales de salud que incluya un componente de promoción de la salud y prevención de las enfermedades. Además, deben asegurar el derecho legal a la confidencialidad y privacidad, establecer servicios de calidad centrados en sus necesidades, e incluir la participación juvenil en el diseño y la prestación de estos servicios.

3. Mejorar el ambiente donde vive el/la adolescente. Los países deben apoyar, fortalecer y preservar a las familias y comunidades a través de su empoderamiento, diseñar políticas y leyes que mejoren las condiciones sociales y económicas de las familias, mejorar los servicios y recursos comunitarios y disminuir la exposición a la violencia y a condiciones y conductas no saludables. También deberán formularse políticas saludables y legislarse (por ejemplo: legislación sobre el tabaco, legislación para la educación secundaria, legislación sobre la edad mínima para contraer matrimonio o legislación relativa al acceso a armas de fuego).

4. Crear normas sociales positivas y comprometerse con la promoción de salud. Los medios de comunicación deben ser parte de la solución, y no el problema, promoviendo mensajes positivos de salud. Se deben crear oportunidades para que se involucre y adopte conductas saludables por medio de información, desarrollo de competencias sociales y habilidades para la vida.

5. Mejorar el acceso a la educación e incrementar el papel de la escuela en la salud de adolescentes. Se deben promover políticas educacionales que aseguren los logros de los estudiantes y que mejoren el vínculo entre escuelas, familias y comunidades. Un desafío importante es disminuir la brecha en el acceso a la educación que existe entre adolescentes rurales y los urbanos y en aquellos de distintos niveles socioeconómicos. También es importante desarrollar políticas integrales de educación que incluyan la salud, estableciendo servicios de salud escolares y un currículum de salud.

6. Apoyar la transición a la edad adulta y promover la inserción laboral. Hay que brindar oportunidades para que los y las adolescentes conozcan las expectativas de los adultos, y aprendan y practiquen papeles adultos en un ambiente positivo, esta- 
bleciendo un puente entre el sistema educativo formal y la estructura productiva. Por ejemplo: apoyar labores de voluntariado y oportunidades en las que adolescentes y jóvenes se sientan miembros activos de sus comunidades. Son prioritarios la mejora de las posibilidades de empleo y el fomento de una independencia futura, así como el mejoramiento del acceso al crédito.

7. Promover un enfoque de género. Es recomendable que los estudios utilicen información desagregada por sexos para conocer más de cerca la situación de mujeres y varones. También se recomienda incorporar el enfoque de género en la capacitación de profesionales, estructurar servicios de salud sensibles a las diferencias de género, promover la equidad y considerar el desarrollo pleno de los seres humanos sin encasillamientos de "masculino" y "femenino".

8. Participación juvenil. Un marco conceptual basado en la promoción de la salud y desarrollo requiere que adolescentes y jóvenes sean los protagonistas de su propia historia y actores estratégicos del desarrollo de sus comunidades y sociedades. Organizaciones sociales de salud, de educación, comunitarias y políticas deben dejar que adolescentes y jóvenes aporten su visión del mundo, sus habilidades sociales y, sobre todo, su energía, para crear una sociedad mejor. La participación juvenil es un elemento clave para el empoderamiento personal y social, una vía de modernización, una forma de aprovechar oportunidades y fortalecer el tejido social.

9. Articulación interinstitucional. Se debe promover esta articulación a escala comunitaria, regional y nacional, con adecuada distribución de roles y funciones, desarrollando programas articulados, focalizados y con protagonismo juvenil.

Para que adolescentes y jóvenes desempeñen un papel importante en nuestras sociedades, hay que asumir la responsabilidad de apoyarlos. Es tiempo de terminar con el silencio político y cultural que existe detrás de la salud de adolescentes y promover su desarrollo pleno.

\section{Referencias}

1. O rganización Panamericana de la Salud. Plan de acción de desarrollo y salud de adolescentes y jóvenes en las A méricas, 1998-2001.W ashington, D.C.: O PS/O MS,1998.
2. Camacho V. Perfil de salud sexual y reproductiva de los y las adolescentes y jóvenes de A mérica Latina y El C aribe: revisión bibliográfica, 19881998. W ashington D.C.: O PS/O MS,2000.

3. Comisión Económica para América Latina y El Caribe. Anuario Estadístico de América Latina y El Caribe 1999. N uevaYork: $N$ aciones Unidas, 2000.

4. O rganización Panamericana de la Salud. La salud del adolescente y el joven. En: La salud en las A méricas. Publicación científica. O PS/O MS,W ashington D.C ., 1998;1:76-81.

5. Comisión Económica paraA mérica Latina y El Caribe. Panorama Social de A mérica Latina 1999-2000. N ueva York: $N$ aciones U nidas, 2000.

6. $O$ rganización de las $\mathrm{N}$ aciones Unidas para la Educación, la Ciencia y la Cultura UN ESC $O$ Statistical Yearbook. Página web de UN ESC 0 , N ueva York: UN ESC 0, 1999.

7. O rganización de las $\mathrm{N}$ aciones Unidas para la Educación, la Ciencia y la Cultura UN ESC 0. Situación educativa deA mérica Latina y El C aribe, 19801994. N ueva York: UN ESC 0, 1996.

8. Comisión Económica para América Latina y El Caribe. Panorama social de América Latina. N ueva York: N aciones U nidas, 1997.

9. O rganización Panamericana de la Salud. Informe anual del Proyecto de Salud Sexual y Reproductiva de Adolescentes y Jóvenes de Centroamérica, W ashington, D.C.: O PS/ASDI, 2000.

10. Burt M. ¿Por qué debemos invertir en el adolescente? Segunda edición. W ashington, D.C.: O PS/O MS, 2000.

11. Schutt-Aine J, Maddaleno M. Salud sexual y desarrollo de adolescentes y jóvenes en las Américas: implicaciones para programas y políticas. W ashington D.C.: O PS/O MS, 2002. En imprenta.

12.A r riagadaV. Familias latinoamericanas: convergencias y divergencias. En Revista CEPAL 1998;(65)28.

13. Fondo de las $\mathrm{N}$ aciones U nidas para la Infancia. The State of Eastern Caribbean Children. Bridgentown, Barbados UN ICEF Caribbean Area O ffice, 1998.

14. Halcón L, Beuhring T, Blum R. A portrait of adolescent health in The Caribbean. Minneapolis, (MN): U niversidad de Minnesota, O PS, 2000.

15. Singh S,W ulf D, Samara R, C ucaY. Gender differences in the timing of first intercourse: D ata from 14 countries. Int Fam Perspect 2000;26.

16. UNAIDS. Joint United Nations Programme on HIV/AIDS Epidemic U pdate. N ueva York UN AIDS, diciembre 1998.

17. Demographic and Health Services. D emographic and health services, 1994-1999. Citado en Progress of N ations. N ueva York: UN ICEF,1999.

18. Espejo F, Sandoval G. Salud mental del adolescente en Latinoamérica y El Caribe.W ashington, D.C.: O PS/O MS, 2002. En prensa.

19. DíazVR. Baja edad de inicio de fumado. En:Diario de la N ación. 2000, noviembre 20, Costa Rica.

20. Morello P, Duggan A, Adger H Jr, Anthony JC, Joffe A. Tobacco use among high school students in BuenosA ires,A rgentina.Am J Public Health 2001;91:219-224.

21. O rganización Panamericana de la Salud. La salud en las Américas. Publicación científica no. 569, 1998;1:193-194.

22. Aguirre R, G üell P, Rojas P, Sáez C. Hacerse hombres: la construcción de la masculinidad en los adolescentes y sus riesgos. W ashington, D.C.: O PS/O MS, 2002. En prensa.

23. Bloom D,W illiamson J. D emographic transitions and economic miracles in emerging A sia. C ambridge (MA): Harvard Institute for International Development, U niversidad de Harvard, 1998.

24. Blum B.Youth and development. Ginebra:W orld Health 0 rganization, 1999.

25. Brindis CD, Irwin CE Jr, O zer EM, Handley M, Knopf DK, Millstein SG. Improving adolescent health: $A n$ analysis and synthesis of health policy recommendations. Resumen del Informe del $\mathrm{N}$ ational Adolescent $\mathrm{Health}$ Information Center, San Francisco (CA): Universidad de California, 1998. 